Anaesthesist 2011 · 60:406

DOI 10.1007/s00101-011-1877-0

Online publiziert: 3. April 2011

(c) Springer-Verlag 2011

\author{
M. Jöhr \\ Institut für Anästhesie, chirurgische Intensivmedizin, Rettungsmedizin \\ und Schmerztherapie, Luzerner Kantonsspital, Luzern
}

\title{
Intubation von Kindern
}

\section{Mit oder ohne Muskelrelaxans?}

Kinder können nach der Gabe von Muskelrelaxanzien intubiert werden, oder auch allein in tiefer Sevofluran- oder Opioid-Propofol-Anästhesie. Beide Vorgehensweisen haben ihre Befürworter und werden in großen Abteilungen praktiziert.

Erstmals wird in Der Anaesthesist das Format der Pro-Kontra-Diskussion verwendet, um Argumente für die eine oder die andere Vorgehensweise aufzuzeigen. Fuchs-Buder et al. [1] befürworten den Einsatz von Muskelrelaxanzien mit klaren und nachvollziehbaren Argumenten; von Ungern-Sternberg [2] lehnt ihn basierend auf großer Erfahrung als Routineverfahren ab.

Die persönliche Haltung des Schreibenden ist, im Sinne einer modernen balancierten Anästhesie zur Intubation in der Regel Relaxanzien zu verwenden; ihre Wirkung wird überwacht und, wenn nötig, antagonisiert. So sind die Intubationsbedingungen zuverlässig gut, auch wenn weniger erfahrene Kollegen beteiligt sind. Pressen oder unerwartetes Bewegen des Patienten wird verhindert, und die Überwachung der Hypnose mithilfe des Elektroenzephalographie- (EEG-) Monitorings gelingt optimiert. Allerdings wird durchaus auch gelegentlich in tiefer Sevoflurannarkose nach einem Bolus von $3 \mathrm{mg} / \mathrm{kgKG}$ Propofol sehr erfolgreich intubiert [3].

Pro-Kontra-Diskussionen spiegeln die klinische Realität oft wenig wider, denn das echte Leben ist nicht einfach schwarzweiß. Sie dienen vielmehr dazu, über die Dinge nachzudenken und die Argumente zu schärfen. Pro-Kontra-Beiträge sind, im Gegensatz zu Übersichten, nicht ab- wägend und ausgeglichen (sie sollen es auch nicht sein), und die Literatur wird ausschließlich zur Stärkung der eigenen Position zitiert. Der Leser soll beide Beiträge lesen, sich eine eigene Meinung bilden, um dann gezielt beim individuellen Patienten das optimale Verfahren zu verwenden. Er wird wahrscheinlich zu dem Schluss kommen, dass letztlich beide Vorgehensweisen in der Kinderanästhesie ihren Platz haben, je nach Erfahrung, Umfeld und klinischer Situation.

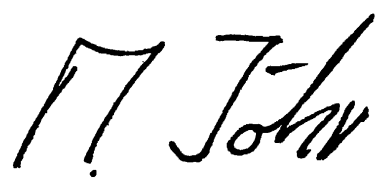

M. Jöhr

\section{Korrespondenzadresse \\ Dr. M. Jöhr \\ Institut für Anästhesie, chirurgische Intensivmedizin, Rettungsmedizin und Schmerztherapie, Luzerner Kantonsspital 6004 Luzern \\ Schweiz \\ joehrmartin@bluewin.ch}

Interessenkonflikt. Der Autor gibt an, dass kein Interessenkonflikt besteht.

\section{Literatur}

1. Fuchs-Buder T, Schreiber JU (2011) Muskelrelaxanzien sind obligat für die Intubation bei Kindern Pro. Anaesthesist 60:474-475

2. von Ungern-Sternberg BS (2011) Muskelrelaxanzien sind obligat für die Intubation bei Kindern Kontra. Anaesthesist 60:476-478

3. Lerman J, Houle TT, Matthews BT et al (2009) Propofol for tracheal intubation in children anesthetized with sevoflurane: a dose-response study. Paediatr Anaesth 19:218-224 